Braz J Med Biol Res, September 2011, Volume 44(9) 890-898

doi: 10.1590/S0100-879X2011007500096

Remodeling in the ischemic heart: the stepwise progression for heart failure

J.G. Mill, I. Stefanon, L. dos Santos and M.P. Baldo

The Brazilian Journal of Medical and Biological Research is partially financed by

\section{虽CNPq}

da Ciência e Tecnologia

Institutional Sponsors
DFAPESP
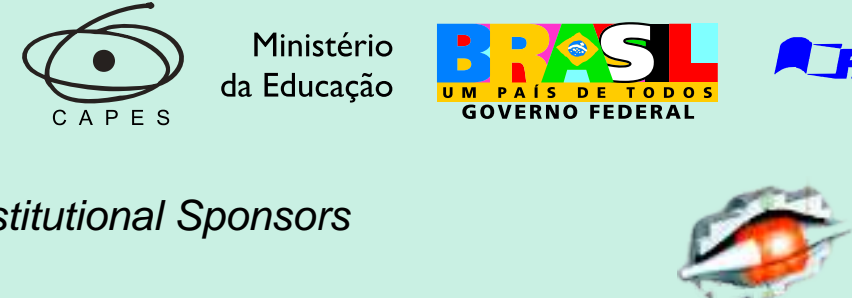

$\sin \theta_{0}$
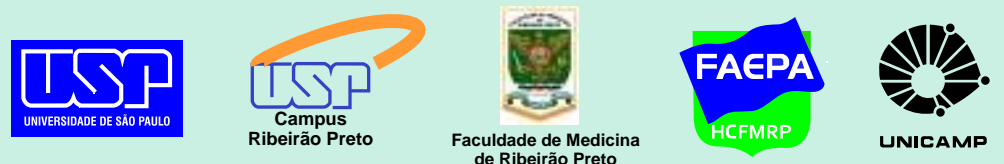

Ф SHIMADZU

Explore High - Performance MS In Prbitrap Technology

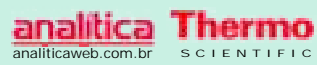




\title{
Remodeling in the ischemic heart: the stepwise progression for heart failure
}

\author{
J.G. Mill, I. Stefanon, L. dos Santos and M.P. Baldo \\ Departamento de Ciências Fisiológicas, \\ Universidade Federal do Espírito Santo, Vitória, ES, Brasil
}

\begin{abstract}
Coronary artery disease is the leading cause of death in the developed world and in developing countries. Acute mortality from acute myocardial infarction (MI) has decreased in the last decades. However, the incidence of heart failure (HF) in patients with healed infarcted areas is increasing. Therefore, HF prevention is a major challenge to the health system in order to reduce healthcare costs and to provide a better quality of life. Animal models of ischemia and infarction have been essential in providing precise information regarding cardiac remodeling. Several of these changes are maladaptive, and they progressively lead to ventricular dilatation and predispose to the development of arrhythmias, HF and death. These events depend on cell death due to necrosis and apoptosis and on activation of the inflammatory response soon after MI. Systemic and local neurohumoral activation has also been associated with maladaptive cardiac remodeling, predisposing to HF. In this review, we provide a timely description of the cardiovascular alterations that occur after $\mathrm{MI}$ at the cellular, neurohumoral and electrical level and discuss the repercussions of these alterations on electrical, mechanical and structural dysfunction of the heart. We also identify several areas where insufficient knowledge limits the adoption of better strategies to prevent HF development in chronically infarcted individuals.
\end{abstract}

Key words: Coronary occlusion; Inflammation; Collagen remodeling; Mechanical dysfunction; Heart failure; Rats

\section{Introduction}

Cardiovascular diseases are the leading cause of death in industrialized countries. These diseases represent a major challenge to the health system because they contribute significantly to healthcare costs and to the degree of disability in the adult population. Stroke and myocardial infarction (MI) are the most important events contributing to this scenario. Survival after acute phase MI has increased in the last decades, mainly due to better management of acute cardiac arrhythmias and the introduction of reperfusion therapies that make it possible to reduce the extent of damage. However, the prevalence of heart failure (HF) is increasing significantly. The post-infarct prevention and treatment of HF have emerged as a growing challenge to improve life quality, increase life expectancy and reduce healthcare costs.

Adequate actions to prevent the development of chronic HF must begin as soon as possible after acute ischemic events because the temporal changes affecting the injured myocardium, such as infarct expansion, myocardial hypertrophy, reactive fibroses, and ventricular dilatation, predispose patients to ventricular failure (Figure 1). An understanding of events occurring at the molecular, cellular and whole tissue level in both the ischemic and surviving area after infarction has been of paramount importance for the development of strategies for better management of patients after coronary occlusion. Moreover, the use of animal models of ischemia/infarction has been essential in providing precise information regarding the cardiac remodeling process.

The knowledge acquired over the last decades from animal models of ischemic HF in parallel to new possibilities provided by genomic manipulation have permitted us to understand the intrinsic mechanisms involved in the progression from ischemic myocardium to HF and to transpose these data to the development of pharmacological and interventional strategies to optimize patient treatment. In this review, our aim is to focus on information gained from animal models of myocardial ischemia about the

Correspondence: M.P. Baldo, Departamento de Ciências Fisiológicas, Universidade Federal do Espírito Santo, Av. Marechal Campos, 1468, 29042-755 Vitória, ES, Brasil. Fax: +55-27-3335-7330. E-mail: marcelobaldo@ymail.com

Presented at the XV Simpósio Brasileiro de Fisiologia Cardiovascular, São Paulo, SP, Brazil, February 2-5, 2011.

Received February 13, 2011. Accepted July 26, 2011. Available online August 5, 2011. Published September 16, 2011. 
cardiovascular alterations that take place in the cellular, neurohumoral and electrical settings during MI. We also discuss the repercussions of these alterations on mechanical and structural dysfunction in the chronic stages of HF, providing a timely description of the mechanisms that underlie the entire myocardial collapse.

\section{Cell death, inflammation and collagen remodeling}

\section{Apoptosis}

As a result of acute coronary occlusion, tissues that receive metabolic supplies from occluded coronary arteries become hypoxic. If ischemia persists, irreversible injury is observed, and cells start to die. In ischemic hearts, cell death occurs through both necrotic and apoptotic pathways, although in the first $24 \mathrm{~h}$ following infarction, apoptosis is the main form of cell death observed (1). Altered apoptosis regulation is due to changes in the expression of pro- and anti-apoptotic proteins. Thus, while the pro-apoptotic protein Bax increases, the anti-apoptotic Bcl-2 decreases. The reduction of the $\mathrm{Bcl}-2 / \mathrm{Bax}$ ratio, a marker of cell viability, is observed as early as $3 \mathrm{~h}$ after coronary occlusion and is associated with high vulnerability of cardiomyocytes to apoptosis (2).

Palojoki et al. (3) observed a high incidence of apoptosis in myocytes in the ischemic border zone the first day after coronary occlusion in rats, which was reduced as the healing process developed (3). However, apoptosis has also been shown to progressively increase in the remote myocardium from the first day to 12 weeks after infarction, as indicated by alterations in some apoptosis-related proteins. This cellular loss may contribute to the progressive decrease of cardiac pumping reserves in the surviving myocardium and subsequent HF.

Apoptosis has great repercussions during the course of cardiac remodeling. Jiang et al. (4) showed a significant relationship between the rate of cardiomyocyte apoptosis, or its regulatory factors, and maladaptive left ventricular remodeling in failing sheep hearts (4). It is not clear if the apoptosis that occurs during myocardial remodeling and $\mathrm{HF}$ is a cause or a consequence of cardiac dysfunction. Nevertheless, apoptosis seems to be an important therapeutic target since it interferes with the infarct area that has long been considered to be the classical determinant of ventricular remodeling and HF progression. Several factors, such as high angiotensin II (Ang II) concentrations and sympathetic overstimulation in infarcted hearts, may contribute to the accelerated rates of apoptosis after infarction. In this context, blockade of the renin-angiotensinaldosterone system (RAAS) may help to preserve myocytes at risk of death. However, no specific therapeutic agent has an anti-apoptotic effect as its main target.

Even though it is well established that the development of HF depends on the size of the scar area (5), our group has

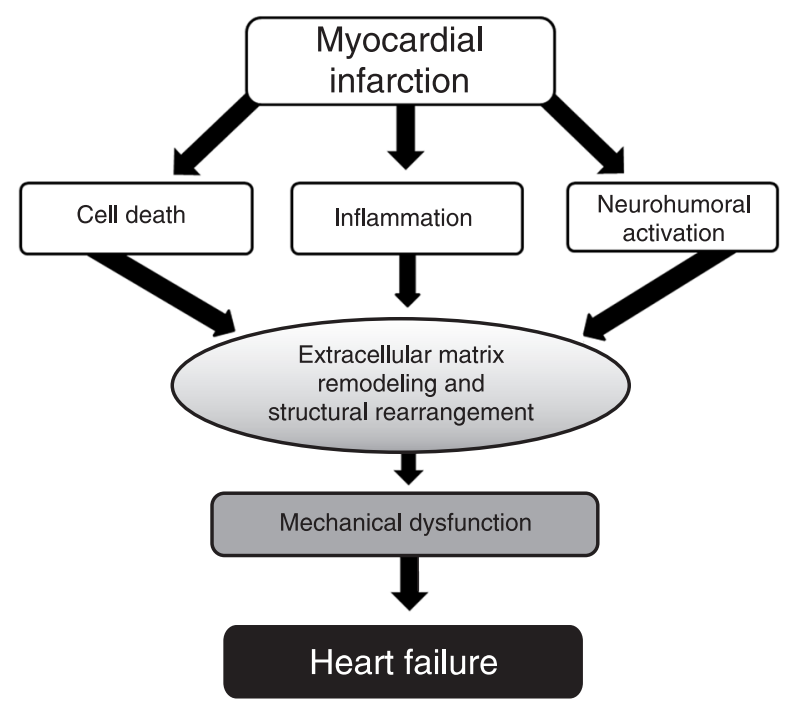

Figure 1. Schematic representation of the time course of postinfarction remodeling.

shown that infarct extent may not be the only, nor perhaps the most important, factor involved in development of HF in the chronic phases of this disease. Rats with similar infarct scar areas do not always develop typical signs of HF (6). In fact, recent studies from our laboratory have shown that rats presenting classical signals of HF around one month after infarction also show reduced water and food intake in the early phase after coronary ligature and different patterns of right and left ventricle extracellular matrix remodeling. These animals also have decreased myocardial contractility but exhibit preserved vascular reactivity $(6,7)$ compared to infarcted rats without HF. Therefore, other predictors of HF development after MI, besides the classic infarct size, can be identified, permitting better prevention strategies.

\section{Inflammation}

The early process of cardiac repair, which includes the mobilization and infiltration of inflammatory cells and collagen breakdown followed by its synthesis, is essential for left ventricular remodeling and must be closely controlled. Although an excessive inflammatory response has deleterious effects on cardiac structure and function, poor prognosis for post-infarction survival is also observed when inflammation is suppressed (8). Thus, this important early phenomenon is an important target for more refined therapeutic interventions since it modulates late cardiac alterations.

When ischemia becomes irreversible and myocytes begin to die, proinflammatory and chemoattractive molecules are released, and inflammatory cells migrate to the ischemic myocardium. These cells are responsible for the phagocytosis of necrotic cells and the initiation of collagen deposition. The cellular response to acute ischemia includes neutrophil, macrophage and lymphocyte infiltration, which 
reaches a peak in the first days and remains elevated until the 4th week post-infarction for the last two immune cell lineages (9). Neutrophils release metalloproteinases (MMP), a large family of enzymes that degrade collagen, while macrophages consume necrotic tissue and release cytokines that stimulate fibroblast proliferation and collagen synthesis. During a persistent ischemic insult, the exposure of the intracellular content to dead cells activates the innate immune system. Although this is the first line of defense against infections, endogenous factors from injured tissue can also stimulate an immune response, such as the activation of Toll-like receptors (TLR). In the heart, TLR2 and TLR4 are perhaps involved in the host response to myocardial infarction. For instance, improved cardiac function and reduced interstitial and perivascular collagen deposition were observed after infarction in both TLR2 knockout (10) and TLR4-defective transgenic mice (11).

Although they are not constitutively expressed in the intact myocardium, several cytokines are intensively synthesized and released in the early period after coronary occlusion and are involved in several cardiac remodeling events such as apoptosis, changes in extracellular matrix and other structural alterations (8). Cytokines such as interleukins (IL)- 6 and $-1 \beta$ and tumor necrosis factor-alpha (TNF- $\alpha$ ) are detected in ischemic tissue as early as $3 \mathrm{~h}$ after the onset of ischemia and are involved in deleterious responses during cardiac remodeling (12). These data show that activation of a local inflammatory response is necessary for tissue healing. However, an exaggerated response seems to be deleterious in the long-term. Therefore, modulation of the inflammatory process in the ischemic and surrounding area may result in preservation of cardiac function in the scarred left ventricle. However, current knowledge regarding the modulation of the inflammatory response after $\mathrm{MI}$ is still insufficient and represents an important area for additional research on experimental models.

\section{Extracellular matrix remodeling}

The postinfarction remodeled myocardium often exhibits increased synthesis and deposition of collagen on the interstitium and on the perivascular space, which contribute to impairment of cardiac function (13).

Collagen synthesis and degradation are closely regulated by several factors, including MMP, tissue inhibitor of matrix metalloproteinase (TIMP), transforming growth factor $\beta$ (TGF- $\beta$ ), and connective tissue growth factor (CTGF). In the acute phase of $\mathrm{MI}$, interstitial collagen is intensely degraded, which contributes to myocyte slippage and wall thinning, critical events in early ventricular expansion and chamber dilatation (14). On the other hand, myocardial profibrotic cytokines, such as TGF- $\beta 1$, are also highly expressed after MI, which induces further conversion of fibroblasts into myofibroblasts, activates gene expression and increases collagen deposition. Expression of CTGF, another profibrotic factor that is also involved in collagen deposition in infarcted hearts, increases in non-infarcted myocardium during injury healing (15). Due to activation of this cytokine cascade, considerable collagen deposition can be observed in infarcted and non-infarcted areas as early as 3 days after MI, which increases during the following 28 days $(16,17)$. Regarding the importance of extracellular matrix remodeling in cardiac dysfunction, Baicu et al. (18) showed that isolated papillary muscles exhibit impaired systolic performance if fibrillar collagen is degraded in a timely manner (18).

Thus, inhibition of MMPs has been proposed as favorable for left ventricle function by accelerating the infarction healing process. Nevertheless, short-term MMP inhibition has positive effects on post-MI remodeling and acute survival, while long-term treatment is associated with cardiac dilatation and dysfunction and increased mortality rate (13). Finally, it is important to point out that common processes closely involved in healing from MI, such as ischemia, inflammation and collagen remodeling, are protagonist targets for HF treatment at the same time. As a result, an understanding of the cardiac repair process is a fundamental step for adequate modulation of MI healing, preservation of cardiac function and long-term survival.

\section{Neurohumoral activation}

\section{Adrenergic activation}

When infarcted hearts display systolic dysfunction, an adaptive neurohumoral response occurs in an attempt to maintain normal cardiac output and systemic blood perfusion. However, if this neurohumoral activation becomes chronic and exaggerated, which basically concerns the sympathetic nervous system (SNS) and the RAAS, the risk for development of HF increases.

The SNS is activated early after MI (19), and its hyperactivity is often associated with contractile dysfunction, cell death, cytokine production, and arrhythmogenesis. On the other hand, cardiac responsiveness to parasympathetic activation seems to be reduced during the remodeling process (20). Systematically, both plasma and tissue levels of catecholamine increase after $\mathrm{MI}$ in rats, and norepinephrine turnover also increases, indicating elevated sympathetic tone in failing hearts. Interestingly, heart rate increases in the acute phase of $\mathrm{MI}$, but is progressively reduced when cardiac dysfunction became evident (19).

Changes in autonomic cardiopulmonary reflexes are time-dependent in post-infarction HF and may contribute to SNS activation. Although in the acute phase both baroreflex and Bezold-Jarisch reflex remain unchanged, in the chronic phase, after coronary occlusion in rats, our group demonstrated impaired cardiopulmonary reflexes when compared to sham-operated animals $(21,22)$. Furthermore, the degree of cardiopulmonary reflex alteration was related to the extent of cardiac necrosis and to the severity of left ventricle dysfunction. 
Another proposed mechanism behind sympathetic hyperactivity after infarction is the activation of the brain RAAS (23). The role of central Ang II in post-myocardial remodeling was indicated by experimental studies showing that blockade of Ang II type 1 receptors $\left(A T_{1}\right)$ in the central nervous system was more effective in preventing cardiac dysfunction than blockade of isolated peripheral $\mathrm{AT}_{1}$ (24). Expression of aldosterone and its receptor increase in the hypothalamus after infarction, which stimulates the production of "ouabain-like" compounds and Ang II, two mechanisms that trigger and amplify the effects of sympathetic activation and predispose to HF. In fact, intracerebroventricular injection of an angiotensin-converting enzyme (ACE) inhibitor improves cardiac function after MI (25).

\section{The cardiac renin-angiotensin-aldosterone system}

The RAAS participates actively in blood pressure regulation and hydroelectrolytic balance. In the last decades, it has been shown that Ang II and other related peptides also have other effects besides the classical ones. These effects are not only mediated by circulating components, but also by paracrine effects of components of the cascade in several organs and tissues, including the heart, blood vessels, kidneys, and central nervous system. It has been described that infarcted rats treated long-term with captopril show hemodynamic preservation and improved survival (26). This classic study highlights the participation of the RAAS cascade in cardiac remodeling.

The cardiac RAAS is activated under several pathophysiological conditions, including MI and HF of different etiologies $(27,28)$. Angiotensinogen is produced in infarcted hearts mainly by macrophages and fibroblasts, and ACE is extensively synthesized in endothelial cells and macrophages (29). ACE activity increases in all regions of the infarcted heart, particularly in the scar tissue, leading to high local Ang II concentrations $(27,30)$. ACE inhibition in infarcted rats reduces mortality, attenuates reactive fibrosis and myocyte hypertrophy and prevents HF development $(16,26,30,31)$. In agreement, expression of $A T_{1}$ receptors is enhanced during the postinfarction period, being highly detected just 3 days after coronary occlusion (29). Moreover, Ang II, acting via AT 1 receptors, elicits local aldosterone production in infarcted hearts. Together, locally produced Ang II and aldosterone are two key mediators of myocyte hypertrophy and enhanced cardiac fibrosis and apoptosis, thus contributing to hemodynamic impairment and HF development (32). In fact, in a mouse model with cardiac overexpression of Ang II, fibrosis was spontaneously observed in transgenic animals, showing that Ang II can act locally independently of hemodynamic effects (33). Moreover, infarction caused an upregulation of mineralocorticoid receptor expression in the left ventricle (30). This increase, which was associated with increased aldosterone production, contributed to harmful collagen proliferation in the overall myocardium, including the right ventricle free wall.

Our knowledge of the RAAS has increased enormously in the last 2 decades after the discovery of the effects of the heptapeptide Ang 1-7, its Mas receptor and the $\mathrm{AT}_{2}$ receptors. An understanding of the effects of these new players on infarct expansion, ventricular remodeling and HF development is still incomplete. However, several studies have shown that Ang II acting on $\mathrm{AT}_{2}$ generally shows opposite effects compared to those related to $A T_{1}$ stimulation. The lack of $A T_{2}$ receptors in infarcted mice led to a higher mortality rate and deleterious effects of ventricular remodeling (34). Moreover, direct $\mathrm{AT}_{2}$ stimulation provides protection from apoptosis and induces an anti-inflammatory response after infarction (35).

As previously stated, collagen proliferation in surviving myocardium negatively affects cardiac function and survival after infarction. Mill et al. (17) showed that aldosterone also contributes to increased collagen deposition in non-infarcted viable tissue. Another effect of aldosterone observed in infarcted rats showed that immediate treatment with eplerenone, a selective aldosterone antagonist, improved left ventricular function and reduced deleterious effects of cardiac remodeling by modulation of inflammatory response in acute phase of the healing process (36). This was mediated by acceleration of macrophage infiltration and necrotic re-absorption. The action of the RAAS in mobilization of inflammatory cells also involves Ang II mediated facilitation of chemokines release, which leads to neutrophil recruitment, as previously described by $\mathrm{Na}-$ bah et al. (37). These effects may contribute to increased neutrophil infiltration into ischemic myocardium, an action that can lead to an abnormal inflammatory response and acute ventricular expansion.

Indeed, regarding remodeling, neurohormonal activation responds to the most important feature of the subacute phase of MI and HF progression. Moreover, it is not difficult to understand why the standard therapy for the treatment of chronic HF includes both ACE inhibitors and $\beta$-blockers as a first line of treatment.

\section{Cellular and mechanical dysfunction in the failing heart}

The myocardial remodeling process occurs at both cellular and subcellular levels, with significant changes seen in the phenotype of every cell comprising the heart tissue. However, more significant changes are seen in the heart's fundamental cell: the cardiomyocyte, which suffers modifications in its size and shape (hypertrophy), and in the molecular mechanisms involved in contraction and relaxation.

\section{Myocyte hypertrophy}

With the loss of functional tissue after MI, the structural and functional remodeling processes occur asymmetrically, 
as characterized by early stretching and thinning of infarcted areas and hypertrophy of the non-infarcted segments, which suffer from increased workload $(1,38)$. While hypertrophy of viable portions of the infarcted heart could be a beneficial compensatory response because it reduces diastolic wall stress, epidemiological studies have shown that hypertrophy is a significant predictor of cardiovascular mortality. In this way, at subsequent stages of cardiac remodeling this event culminates in contractile failure and ventricular dilatation.

Cardiomyocyte hypertrophy is a complex process that occurs in response to mechanical overload as an adaptive mechanism to improve pumping function by increasing the amount of contractile units and reducing wall stress (39). It is characterized primarily by increased cell size, increased sarcomeres and reorganization of the intracellular components, which depend on intracellular regulation of protein synthesis. Although mechanical factors are the most important stimulus, other factors such as hormones, cytokines and intracellular pathways also play a key role in the hypertrophic process. These other hypertrophic stimuli include adrenergic activation, Ang II, endothelin-1, thyroid hormone, insulin, cytokines, IGF (insulin-like growth factors) I and II, and even membrane receptors classified as stretch-sensitive receptors (40). These substances act at specific membrane receptors to mobilize multiple intracellular signaling pathways through intermediate molecules and factors responsible for transcriptional regulation of gene expression. The major signaling moduluses involved in the cardiomyocyte hypertrophic response are mitogen activated protein kinase (MAPK), PI3K-Akt, and calcineurin-NFAT. Activation of a cascade comprised of the MAPK superfamily has been shown to play a close causal role in hypertrophy and in the transition to HF (41). The phospholipid kinase PI3K has hypertrophic effects on the cardiomyocyte mediated by Akt and can activate protein synthesis and also produce cytoprotective effects. Finally, the calcineurin-NFAT pathway is also an important signaling cascade involved in cardiac hypertrophy that indirectly regulates the transcription of pro-hypertrophic genes in infarcted hearts (42).

\section{Molecular substrate for contractile dysfunction}

Changes in several molecules related to contractile function of the cardiomyocyte are also associated with hypertrophy. Even before the organism presents signs of HF, myocardial contractility is reduced due to post-MI remodeling that results from alterations in key cytoplasmic and membrane proteins involved in the contraction-relaxation cycle $(43,44)$. There are basically two subcellular defects that may contribute to the development of myocardial dysfunction: deranged calcium handling and/or alteration of myofilament responsiveness. The first mechanism seems to be more important in contributing to changes in excitation-contraction coupling in infarcted hearts.

Calcium is important for many crucial intracellular functions in cardiomyocytes, such as regulation of energy metabolism, protein synthesis, and especially for coupling between excitation and contraction. In fact, the most studied mechanism for contractile dysfunction in post-infarction remodeled myocardium is defective calcium handling. Impairment in primary transsarcolemmal $\mathrm{Ca}^{2+}$ influx, $\mathrm{Ca}^{2+}$-induced $\mathrm{Ca}^{2+}$ release, and $\mathrm{Ca}^{2+}$ re-uptake by the sarcoplasmic reticulum (SR) use to be the main mechanisms for regulating levels of the molecular substrates for the depressed contraction and relaxation processes (45). In a rat model of MI, our group was one of the first to show that remodeled cardiac muscle exhibits depressed post-rest potentiation of contraction (46), which reinforced the current idea that the SR is functionally impaired. Therefore, we are currently investigating the exact contributions of these factors by using molecular biology tools.

Primary transsarcolemmal $\mathrm{Ca}^{2+}$ influx leads to $\mathrm{Ca}^{2+}$ release by the junctional SR for use during contraction. During the compensated stage of cardiac remodeling both sarcolemmal L-type $\mathrm{Ca}^{2+}$ channel density and $I_{\mathrm{C}, \mathrm{L}}$ are unchanged; however, in advanced stages of HF, these parameters are reduced to some extent in infarcted hearts (47). The SR is the major source of the activator $\mathrm{Ca}^{2+}$ for contraction. The $\mathrm{Ca}^{2+}$ release channels of the $\mathrm{SR}$ are part of a complex molecular structure known as the ryanodine receptor (RyR, or more accurately, RyR2 for the cardiac isoform) and the minor, but important, inositol 1,4,5-triphosphate sensible receptor (IP3 receptor). After MI, a decline in the mRNA levels and density of RyR2 occurs, which has been described in studies with experimental models (48).

Although a different arrangement of myosin and actin is seen due to the expression of different isoforms or post-transcriptional reorganization, the isoform pattern is substantially specific for each species, organ and pathological condition. After birth, the a-type myosin heavy chain $(\alpha-M H C)$, which has a higher ATPase activity and shortening velocity, is predominant in the ventricles while $\beta-\mathrm{MHC}$ is suppressed (49). However, several stimuli are able to modify this transcriptional pattern, with $\beta-\mathrm{MHC}$ gene expression often being induced together with other genes of the fetal program during cellular remodeling. As a result, myocytes exhibit slower shortening velocity and impaired relaxation, which could contribute, at least in part, to contractile dysfunction (49). During relaxation, most of the cytosolic $\mathrm{Ca}^{2+}$ is rapidly taken up by $\mathrm{SR}$ vesicles, and a smaller amount is secreted through the sarcolemma membrane by a membrane anchored ATPase. Thus, SR dysfunction found in the post-MI remodeled heart is characterized by an imbalance between uptake, storage and release of $\mathrm{Ca}^{2+}$ to the myoplasma. $\mathrm{Ca}^{2+}$ uptake by the $\mathrm{SR}$ is reduced in remodeled myocardium and is mainly the result of reduced levels of sarcoplasmic reticulum $\mathrm{Ca}^{2+}$-ATPase (SERCA-2) (48), the ATPase responsible for pumping this ion into the SR. SERCA-2 overexpression in a porcine model of ischemia and reperfusion was able to attenuate arrhythmias, mainly in the reperfusion period (50). Therefore, improve- 
ment in calcium handling may reduce arrhythmias in the ischemic myocardium. However, the impaired re-uptake of $\mathrm{Ca}^{2+}$ is not only due to decreased SERCA-2 but also to the lower phosphorylation status of its regulatory protein phospholamban (51).

As mentioned before, during relaxation, $\mathrm{Ca}^{2+}$ must be taken up by the SR or secreted into the extracellular space by the sarcolemmal sodium-calcium exchanger (NCX) and the sarcolemmal $\mathrm{Ca}^{2+}$ pump ( $\mathrm{Ca}^{2+}$-ATPase). Few studies have focused on the activity or expression of the sarcolemmal $\mathrm{Ca}^{2+}$ ATPase during cardiac remodeling, but it is believed that they remain unchanged despite several modifications in cardiomyocyte phenotype (52). On the other hand, there is a consensus that NCX protein content and activity can increase during the final stages of remodeling and during the transition from compensated to uncompensated HF in experimental animals (53). Although an exact profile of the quantity and activity of these calcium handling and contractile proteins may be controversial and dependent on the cause and stage of myocardial remodeling, the importance of this intracellular milieu in contractile dysfunction in $\mathrm{HF}$ is unquestionable. It is still unclear whether the changes in calcium handling in cardiomyocytes are a cause or consequence of HF. Longitudinal studies may provide information on the temporal relationship between these processes.

\section{Electrophysiological changes and arrhythmogenesis}

Ventricular tachyarrhythmias are the main cause of death following coronary occlusion in rats (54) (Figure 2). Within the first 24-h of coronary occlusion, the incidence of ventricular arrhythmias increases with high severity, and ventricular tachycardia and fibrillation are frequently observed (55). Phase 1 arrhythmias are initiated approximately 2 min after coronary occlusion and remain until approximately 20 min later (54). Arrhythmias of the first phase are mainly due to disturbances in resting membrane potential, while electrical uncoupling is mostly involved in those occurring later on. Acute membrane lesions and exposure of intracellular content lead to potassium accumulation in the extracellular clefts between myocyte strands and to reduction in the resting membrane potential, which interferes with the generation and conduction of the electrical stimulus through the ventricle. In contrast to phase 1, the mechanisms involved in the development and maintenance of phase 2 of arrhythmias are poorly understood. Coronary occlusion in anesthetized or awake, freely moving animals leads to a large incidence of ventricular tachyarrhythmias from 90 $\min$ to $12 \mathrm{~h}$ post-occlusion (55). An important observation is that phase 2 ventricular arrhythmias are absent in isolated perfused hearts subjected to coronary occlusion, calling attention to blood components and autonomic modulation as initiators of ventricular arrhythmias. While some hypoth-

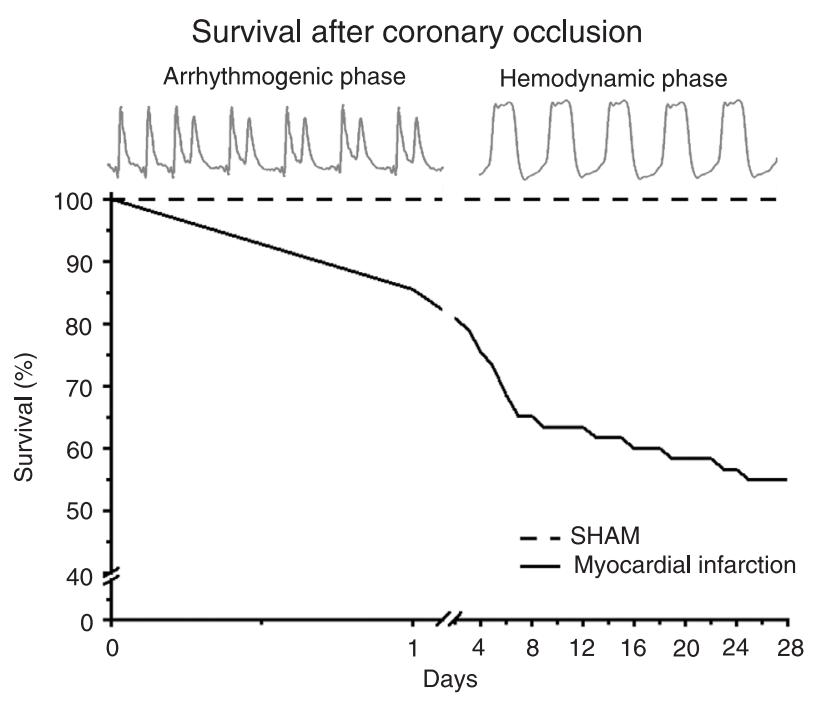

Figure 2. Distinct phases of mortality after myocardial infarction during 28 days. Kaplan-Meier curves show an initial phase (first $24 \mathrm{~h}$ ) in which arrhythmias are the main cause of death. The second period corresponds to those animals that die by cardiac failure.

eses have been tested, the mechanism underlying these late arrhythmias remains unknown. However, a complex mechanism involving adrenergic activation and neutrophil engraftment was recently proposed to explain delayed arrhythmogenesis (56). Together, phases 1 and 2 ventricular arrhythmias are coincident with mortality periods during the first 24-h after myocardial infarction (55).

After this early arrhythmogenic period, ventricular remodeling affects the electrical properties of the myocardium, generating electrical instability that can predispose to ventricular fibrillation and sudden cardiac death. Changes in ion channel expression and function occur either in the peri-infarct area or in remote regions of the scar tissue (57). These changes, named electrical remodeling, produce an electrical gradient during repolarization between infarcted areas, the ischemic zone and viable myocardium that may contribute to re-entrant arrhythmias (58).

Three days after coronary occlusion, reduction of potassium currents $\left(I_{\mathrm{k}}, I_{\text {to }}\right.$ and $\left.I_{\mathrm{k} 1}\right)$ can be observed in the left ventricle, showing differences between endocardial and epicardial layers $(59,60)$. Moreover, the expression of some subunits of potassium channels remains reduced in infarcted rats, which affects the repolarization process. Because this process is not homogeneous among all myocardium that survive infarction (57), the repolarization process can become asynchronous in different parts of the left ventricle, increasing susceptibility to development of ventricular arrhythmias (59). These early alterations are dissociated from structural remodeling and help to explain the enhanced duration of action potentials observed in 
myocytes early after infarction (60). Although the most common mechanisms underlying ventricular arrhythmias are the re-entrant circuits, a non-reentrant mechanism can also lead to electrical instability in the myocardium. Intracellular calcium overload, as seen after infarction, may predispose one to the appearance of ectopic depolarization before complete repolarization (58). From an electrophysiological view, it has been proposed that alterations in expression and functionality of calcium handling proteins may favor electrical instability in failing hearts. However, the actual level of knowledge about the mechanisms involved in arrhythmogenesis in remodeled hearts exhibiting signs of HF is still insufficient for the adequate handling of patients with complex arrhythmias, mainly those associated with precocious ventricular premature beats, which frequently evolve to ventricular tachycardia and fibrillation. Sudden cardiac death is still a frequent event in patients with HF, and a better understanding of arrhythmogenesis in experimental models of this disease is necessary to reduce fatal events in this condition.

\section{Future directions}

Based on the pathogenesis of post-infarct left ventricular remodeling presented in this review, we can suggest some ideas concerning HF therapy. Current therapy for HF is based on neurohormonal blockade by renin-angiotensinaldosterone blockers (i.e., ACE inhibitors, $\mathrm{AT}_{1}$ antagonists, aldosterone receptor blockers) and $\beta$-blockers. These drugs have been shown to have a great impact on post-infarction survival rates over the last three decades. This fact emphasizes the real importance of neurohumoral activation on ventricular remodeling. However, new candidate therapies are focusing on early events. For example, TNF- $\alpha$ inhibitors have been tested, and erythropoietin and granulocyte colony-stimulating factor (G-CSF) appear to be interesting adjuvant options. Moreover, despite the fact that collagen reduction is a target for therapy, some studies have proposed that inhibition of MMPs in the acute phase of infarction could be an important therapeutic target.

In summary, a perfect understanding of post-infarct left ventricular remodeling kinetics will allow for the development of effective therapeutic panels for reducing the growing epidemic of HF and its associated mortality.

\section{Acknowledgments}

We acknowledge the continuous support of CNPq and Fundação de Amparo à Pesquisa do Espírito Santo (FAPES).

\section{References}

1. dos Santos L, Santos AA, Goncalves GA, Krieger JE, Tucci PJ. Bone marrow cell therapy prevents infarct expansion and improves border zone remodeling after coronary occlusion in rats. Int J Cardiol 2010; 145: 34-39.

2. Simonis G, Wiedemann S, Schwarz K, Christ T, Sedding DG, $\mathrm{Yu} X$, et al. Chelerythrine treatment influences the balance of pro- and anti-apoptotic signaling pathways in the remote myocardium after infarction. Mol Cell Biochem 2008; 310: 119-128.

3. Palojoki E, Saraste A, Eriksson A, Pulkki K, Kallajoki M, Voipio-Pulkki LM, et al. Cardiomyocyte apoptosis and ventricular remodeling after myocardial infarction in rats. $A m ~ J$ Physiol Heart Circ Physiol 2001; 280: H2726-H2731.

4. Jiang L, Huang Y, Hunyor S, dos Remedios CG. Cardiomyocyte apoptosis is associated with increased wall stress in chronic failing left ventricle. Eur Heart $J$ 2003; 24: 742-751.

5. Pfeffer MA, Pfeffer JM, Fishbein MC, Fletcher PJ, Spadaro $\mathrm{J}$, Kloner RA, et al. Myocardial infarct size and ventricular function in rats. Circ Res 1979; 44: 503-512.

6. Pereira RB, Sartorio CL, Vassallo DV, Stefanon I. Differences in tail vascular bed reactivity in rats with and without heart failure following myocardial infarction. $J$ Pharmacol Exp Ther 2005; 312: 1321-1325.

7. Sartorio CL, Fraccarollo D, Galuppo P, Leutke M, Ertl G, Stefanon I, et al. Mineralocorticoid receptor blockade improves vasomotor dysfunction and vascular oxidative stress early after myocardial infarction. Hypertension 2007; 50: 919925.

8. Frangogiannis NG, Smith CW, Entman ML. The inflam- matory response in myocardial infarction. Cardiovasc Res 2002; 53: 31-47.

9. Jourdan-Lesaux C, Zhang J, Lindsey ML. Extracellular matrix roles during cardiac repair. Life Sci 2010; 87: 391-400.

10. Shishido T, Nozaki N, Yamaguchi S, Shibata Y, Nitobe J, Miyamoto T, et al. Toll-like receptor-2 modulates ventricular remodeling after myocardial infarction. Circulation 2003; 108: $2905-2910$

11. Timmers L, Sluijter JP, van Keulen JK, Hoefer IE, Nederhoff MG, Goumans MJ, et al. Toll-like receptor 4 mediates maladaptive left ventricular remodeling and impairs cardiac function after myocardial infarction. Circ Res 2008; 102: 257-264.

12. Deten A, Volz HC, Briest W, Zimmer HG. Cardiac cytokine expression is upregulated in the acute phase after myocardial infarction. Experimental studies in rats. Cardiovasc Res 2002; 55: 329-340.

13. Spinale FG. Myocardial matrix remodeling and the matrix metalloproteinases: influence on cardiac form and function. Physiol Rev 2007; 87: 1285-1342.

14. Whittaker P, Boughner DR, Kloner RA. Role of collagen in acute myocardial infarct expansion. Circulation 1991; 84: 2123-2134.

15. Dean RG, Balding LC, Candido R, Burns WC, Cao Z, Twigg $\mathrm{SM}$, et al. Connective tissue growth factor and cardiac fibrosis after myocardial infarction. J Histochem Cytochem 2005; 53: $1245-1256$.

16. Milanez MC, Gomes MG, Vassallo DV, Mill JG. Effects of captopril on interstitial collagen in the myocardium after 
infarction in rats. J Card Fail 1997; 3: 189-197.

17. Mill JG, Milanez MC, de Resende MM, Gomes MG, Leite $\mathrm{CM}$. Spironolactone prevents cardiac collagen proliferation after myocardial infarction in rats. Clin Exp Pharmacol Physiol 2003; 30: 739-744.

18. Baicu CF, Stroud JD, Livesay VA, Hapke E, Holder J, Spinale $\mathrm{FG}$, et al. Changes in extracellular collagen matrix alter myocardial systolic performance. Am J Physiol Heart Circ Physiol 2003; 284: H122-H132.

19. Mill JG, Vassallo DV, Leite CM. Time course of changes in heart rate and sympathetic tone after coronary artery ligation in rats. Braz J Med Biol Res 1991; 24: 855-858.

20. Modolo RP, Bernardes CF, Vasquez EC, Mill JG. [Evaluation of the vagal efferent pathway in rats in the acute and chronic phases of myocardial infarction]. Arq Bras Cardiol 1995; 65: 17-22.

21. Meyrelles SS, Mill JG, Cabral AM, Vasquez EC. Cardiac baroreflex properties in myocardial infarcted rats. $J$ Auton Nerv Syst 1996; 60: 163-168.

22. Meyrelles SS, Bernardes CF, Modolo RP, Mill JG, Vasquez EC. Bezold-Jarisch reflex in myocardial infarcted rats. $J$ Auton Nerv Syst 1997; 63: 144-152.

23. Westcott KV, Huang BS, Leenen FH. Brain renin-angiotensin-aldosterone system and ventricular remodeling after myocardial infarct: a review. Can J Physiol Pharmacol 2009; 87: 979-988.

24. Huang BS, Ahmad M, Tan J, Leenen FH. Chronic central versus systemic blockade of AT(1) receptors and cardiac dysfunction in rats post-myocardial infarction. Am J Physiol Heart Circ Physiol 2009; 297: H968-H975.

25. Araujo IG, Trindade DC, Mecawi AS, Sonoda-Cortes R, Werneck-de-Castro JP, Costa-E-Sousa RH, et al. Inhibition of brain renin-angiotensin system improves diastolic cardiac function following myocardial infarction in rats. Clin Exp Pharmacol Physiol 2009; 36: 803-809.

26. Pfeffer MA, Pfeffer JM, Steinberg C, Finn P. Survival after an experimental myocardial infarction: beneficial effects of longterm therapy with captopril. Circulation 1985; 72: 406-412.

27. Busatto VC, Cicilini MA, Mill JG. Increased angiotensinconverting enzyme activity in the left ventricle after infarction. Braz J Med Biol Res 1997; 30: 679-687.

28. Resende MM, Mill JG. Alternate angiotensin Il-forming pathways and their importance in physiological or physiopathological conditions. Arq Bras Cardiol 2002; 78: 425-438.

29. Sun Y, Weber KT. Angiotensin converting enzyme and myofibroblasts during tissue repair in the rat heart. $J$ Mol Cell Cardiol 1996; 28: 851-858.

30. de Resende MM, Kauser K, Mill JG. Regulation of cardiac and renal mineralocorticoid receptor expression by captopril following myocardial infarction in rats. Life Sci 2006; 78: 3066-3073.

31. Mill JG, Gomes AP, Carrara AB, Gomes MG, Vassallo DV. Influence of chronic captopril therapy on the mechanical performance of the infarcted rat heart. Pharmacol Res 1994; 29: 77-88.

32. Paul M, Poyan MA, Kreutz R. Physiology of local reninangiotensin systems. Physiol Rev 2006; 86: 747-803.

33. Xu J, Carretero OA, Lin CX, Cavasin MA, Shesely EG, Yang $\mathrm{JJ}$, et al. Role of cardiac overexpression of ANG II in the regulation of cardiac function and remodeling postmyocardial infarction. Am J Physiol Heart Circ Physiol 2007; 293: $\mathrm{H} 1900-\mathrm{H} 1907$.
34. Adachi Y, Saito Y, Kishimoto I, Harada M, Kuwahara K, Takahashi N, et al. Angiotensin II type 2 receptor deficiency exacerbates heart failure and reduces survival after acute myocardial infarction in mice. Circulation 2003; 107: 24062408.

35. Kaschina E, Grzesiak A, Li J, Foryst-Ludwig A, Timm M, Rompe $\mathrm{F}$, et al. Angiotensin II type 2 receptor stimulation: a novel option of therapeutic interference with the reninangiotensin system in myocardial infarction? Circulation 2008; 118: 2523-2532.

36. Fraccarollo D, Galuppo P, Schraut S, Kneitz S, van Rooijen $\mathrm{N}$, Ertl G, et al. Immediate mineralocorticoid receptor blockade improves myocardial infarct healing by modulation of the inflammatory response. Hypertension 2008; 51: 905-914.

37. Nabah YN, Mateo T, Estelles R, Mata M, Zagorski J, Sarau $\mathrm{H}$, et al. Angiotensin II induces neutrophil accumulation in vivo through generation and release of CXC chemokines. Circulation 2004; 110: 3581-3586.

38. dos Santos L, Antonio EL, Souza AF, Tucci PJ. Use of afterload hemodynamic stress as a practical method for assessing cardiac performance in rats with heart failure. Can J Physiol Pharmacol 2010; 88: 724-732.

39. Fedak PW, Verma S, Weisel RD, Li RK. Cardiac remodeling and failure: from molecules to man (Part I). Cardiovasc Pathol 2005; 14: 1-11.

40. Gradman AH, Alfayoumi F. From left ventricular hypertrophy to congestive heart failure: management of hypertensive heart disease. Prog Cardiovasc Dis 2006; 48: 326-341.

41. Clerk A, Sugden PH. Activation of protein kinase cascades in the heart by hypertrophic $\mathrm{G}$ protein-coupled receptor agonists. Am J Cardiol 1999; 83: 64H-69H.

42. Wilkins BJ, Molkentin JD. Calcium-calcineurin signaling in the regulation of cardiac hypertrophy. Biochem Biophys Res Commun 2004; 322: 1178-1191.

43. Mill JG, Stefanon I, Leite CM, Vassallo DV. Changes in performance of the surviving myocardium after left ventricular infarction in rats. Cardiovasc Res 1990; 24: 748-753.

44. Stefanon I, Auxiliadora-Martins M, Vassallo DV, Mill JG. Analysis of right and left ventricular performance of the rat heart with chronic myocardial infarction. Braz J Med Biol Res 1994; 27: 2667-2679.

45. Bers DM. Altered cardiac myocyte Ca regulation in heart failure. Physiology 2006; 21: 380-387.

46. Novaes MA, Stefanon I, Mill JG, Vassallo DV. Contractility changes of the right and ventricular muscle after chronic myocardial infarction. Braz J Med Biol Res 1996; 29: 16831690.

47. Kim SJ, Kudej RK, Yatani A, Kim YK, Takagi G, Honda R, et al. A novel mechanism for myocardial stunning involving impaired $\mathrm{Ca}^{2+}$ handling. Circ Res 2001; 89: 831-837.

48. Sallinen P, Manttari S, Leskinen H, Ilves M, Ruskoaho H, Saarela $S$. Time course of changes in the expression of DHPR, RyR(2), and SERCA2 after myocardial infarction in the rat left ventricle. Mol Cell Biochem 2007; 303: 97-103.

49. Palmer BM. Thick filament proteins and performance in human heart failure. Heart Fail Rev 2005; 10: 187-197.

50. Prunier F, Kawase Y, Gianni D, Scapin C, Danik SB, Ellinor $\mathrm{PT}$, et al. Prevention of ventricular arrhythmias with sarcoplasmic reticulum $\mathrm{Ca}^{2+}$ ATPase pump overexpression in a porcine model of ischemia reperfusion. Circulation 2008; 118: 614-624.

51. Sande JB, Sjaastad I, Hoen IB, Bokenes J, Tonnessen T, 
Holt E, et al. Reduced level of serine(16) phosphorylated phospholamban in the failing rat myocardium: a major contributor to reduced SERCA2 activity. Cardiovasc Res 2002; 53: 382-391.

52. Dixon IM, Hata T, Dhalla NS. Sarcolemmal calcium transport in congestive heart failure due to myocardial infarction in rats. Am J Physiol 1992; 262: H1387-H1394.

53. Gomez AM, Schwaller B, Porzig H, Vassort G, Niggli E, Egger M. Increased exchange current but normal $\mathrm{Ca}^{2+}$ transport via $\mathrm{Na}^{+}-\mathrm{Ca}^{2+}$ exchange during cardiac hypertrophy after myocardial infarction. Circ Res 2002; 91: 323-330.

54. Baldo MP, Davel AP, Nicoletti-Carvalho JE, Bordin S, Rossoni LV, Mill JG. Granulocyte colony-stimulating factor reduces mortality by suppressing ventricular arrhythmias in acute phase of myocardial infarction in rats. $J$ Cardiovasc Pharmacol 2008; 52: 375-380.

55. Opitz CF, Mitchell GF, Pfeffer MA, Pfeffer JM. Arrhythmias and death after coronary artery occlusion in the rat. Con- tinuous telemetric ECG monitoring in conscious, untethered rats. Circulation 1995; 92: 253-261.

56. Clements-Jewery H, Andrag E, Hearse DJ, Curtis MJ. Complex adrenergic and inflammatory mechanisms contribute to phase 2 ventricular arrhythmias in anaesthetized rats. $\mathrm{Br} \mathrm{J}$ Pharmacol 2009; 156: 444-453.

57. Santos PE, Barcellos LC, Mill JG, Masuda MO. Ventricular action potential and L-type calcium channel in infarctinduced hypertrophy in rats. J Cardiovasc Electrophysiol 1995; 6: 1004-1014.

58. Antoons G, Sipido KR. Targeting calcium handling in arrhythmias. Europace 2008; 10: 1364-1369.

59. Yao JA, Jiang M, Fan JS, Zhou YY, Tseng GN. Heterogeneous changes in $\mathrm{K}$ currents in rat ventricles three days after myocardial infarction. Cardiovasc Res 1999; 44: 132-145.

60. Huang B, Qin D, El-Sherif N. Early down-regulation of $\mathrm{K}^{+}$ channel genes and currents in the postinfarction heart. $J$ Cardiovasc Electrophysiol 2000; 11: 1252-1261. 